\title{
Toward an Autonomous Lunar Landing Based on Low-speed Optic Flow Sensors
}

\author{
Guillaume Sabiron, Paul Chavent, Laurent Burlion, Erwan Kervendal, Eric \\ Bornschlegl, Patrick Fabiani, Thibaut Raharijaona and Franck Ruffier
}

\begin{abstract}
For the last few decades, growing interest has returned to the quite challenging task of the autonomous lunar landing. Soft landing of payloads on the lunar surface requires the development of new means of ensuring safe descent with strong final conditions and aerospace-related constraints in terms of mass, cost and computational resources. In this paper, a two-phase approach is presented: first a biomimetic method inspired from the neuronal and sensory system of flying insects is presented as a solution to perform safe lunar landing. In order to design an autopilot relying only on optic flow (OF) and inertial measurements, an estimation method based on a two-sensor setup is introduced: these sensors allow us to accurately estimate the orientation of the velocity vector which is mandatory to control the lander's pitch in a quasi-optimal way with respect to the fuel consumption. Secondly a new low-speed Visual Motion Sensor (VMS) inspired by insects' visual systems performing local angular 1-D speed measurements ranging from $1.5^{\circ} / \mathrm{s}$ to $25^{\circ} / \mathrm{s}$ and weighing only $2.8 \mathrm{~g}$ is presented. It was tested under free-flying outdoor conditions over various fields onboard an $80 \mathrm{~kg}$ unmanned helicopter. These preliminary results show that the optic flow measured despite the complex disturbances encountered closely matched the ground-truth optic flow.
\end{abstract}

Guillaume Sabiron · Thibaut Raharijaona · Franck Ruffier

Aix-Marseille Université, Biorobotic Dept. of ISM, CNRS, ISM UMR 7287, 13288, Marseille cedex 09, France

e-mail: \{Guillaume.Sabiron,Thibaut.Raharijaona,Franck.Ruffier\}@univ-amu.fr.

Guillaume Sabiron · Paul Chavent · Laurent Burlion · Patrick Fabiani

ONERA - The French Aerospace Lab, F-31055 Toulouse, France

e-mail: \{Guillaume.Sabiron, Paul.Chavent, Laurent.Burlion, Patrick.Fabiani\}@ onera.fr

Erwan Kervendal

Astrium Satellites, Toulouse, France e-mail: Erwan.Kervendal@astrium.eads.net

Eric Bornschlegl

European Space Agency ESTEC, 2200 AG Noordwijk, The Netherlands

e-mail: Eric.Bornschlegl@esa.int 


\section{Introduction}

Landing on extraterrestrial bodies is still a critical stage in any exploration missions. A safe and soft landing is therefore required even though the task is way harder than on the Earth. On the Moon, the lack of atmosphere (acting as a natural brake or allowing the use of a parachute as on Mars [6]) and also the lack of usual sensing systems such as Global Positioning System (GPS) increase the complexity. On top of that, the time delay introduced by Earth to Moon communications forces the landing to be autonomous and based on robust and reliable sensors. Furthermore, sharp constraints in terms of embedded mass led us to design a very lightweight biologically inspired sensor that measures the visual angular velocity (in \% $\%$ ) of the images sweeping backward across the visual field which is known as the optic flow. The visual cues seem to be a promising way to achieve autonomous lunar landing. Recently, several studies have shown various visual techniques such as LIDAR (LIght Detection And Ranging) techniques [36,37] or other vision based navigation systems to estimate position and velocity parameters $[7,11,16,25,34,41,47,50]$, to perform hazard avoidance [48] or to control unmanned spacecraft [23, 24, 51]. In [51], the optic flow regulation principle [45] was applied to autonomous lunar landing problems using a feedback loop and tested by performing simulations on PANGU software (Planet and Asteroid Natural scene Generation Utility) developed for ESA by the University of Dundee which is a tool used to simulate visual environment of planetary surfaces(see [9,38] for more information). In [24], based on numerical simulations, optimal trajectories were calculated in terms of the duration of the landing phase or the fuel consumption while keeping the OF constant. In [32], a fully optic flow-based visual servo control system was developed, in which a large visual field was combined with a centroid in order to estimate the direction of the speed vector in the case of small aerial robotic vehicles. In the extended Kalman filter (EKF) method described in [34], both of the above visual approaches were combined with an Inertial Measurement Unit (IMU), and accurate estimates of the lander's terrain-relative position, attitude, and velocity were obtained. In the preliminary strategy presented here, we focus on means to estimate from optic flow signals useful information such as the orientation of the velocity vector. Finding means of sensing the optic flow onboard unmanned aerial and terrestrial vehicles has been a key research topic during the last few decades. Several flight control systems based on optic flow cues have been constructed so far for performing hazardous tasks such as hovering and landing on a moving platform [21], avoiding obstacles [1, 3, 20], following terrain [18] and tracking a moving target [28]. Insects are capable of impressive flying behavior thanks to the built-in abilities they have developed and improved over several hundred millions of years, despite their small size and hence limited neural resources. Based on the findings obtained at our Laboratory on the fly's visual sensory system [12], several versions of the 2-pixel Local Motion Sensor (LMS) [10, 13, 14, 44,46] were developed, using an algorithm introduced by [5,39], which was later called the "time of travel scheme" (see [2,33]). Several visionbased systems have been previously designed to measure the optic flow onboard UAVs (Unmanned Aerial Vehicles) $[8,19,22]$ and in particular in the range expe- 
rienced during lunar landing $[20,28,52]$. Most of these visual systems were quite demanding in terms of their computational requirements and/or their weight or were not very well characterized, except for the optical mouse sensors [4], with which a standard error of approximately $\pm 5^{\circ} / \mathrm{s}$ around $25^{\circ} / \mathrm{s}$ was obtained in the case of an optical mouse sensor measuring motion in a $\pm 280^{\circ} / \mathrm{s}$ overall range. However, to our knowledge, very few studies have been published so far in which optic flow systems have been implemented and tested outdoors onboard an unmanned aircraft subject to vibrations, where the illuminance cannot be easily controlled (see [1] in the case of linear 1-D motion sensors and see [18, 20,27,49] in that of 2-D optic flow sensors). A particular effort has been made in this study to cope the sensor's measurement range $\left[1.5^{\circ} / \mathrm{s} ; 25^{\circ} / \mathrm{s}\right]$ with the one experienced during a lunar landing approach phase approximately of $\left[2^{\circ} / \mathrm{s} ; 6^{\circ} / \mathrm{s}\right]$. It therefore seemed to be worth testing the reliability of the present 1-D optic flow-based visual sensor on a platform featuring the most similar conditions as on a spacecraft during lunar landing in terms of vibration dynamics and optic flow measurement range. Finally the sensor has been embedded onboard a free-flying helicopter called ReSSAC (ReSSAC stands in French for Recherche et Sauvetage par Système Autonome Coopérant) and tested in terms of its resolution, accuracy, sensitivity over a series of trees. This paper is organized as follows. The intended control strategy, the reference descent trajectory and the basic equations of the lunar lander dynamics allowing the estimation of the orientation of the velocity vector are described in Sect. 2. Section 3 gives a brief description of the new 1-D visual motion device, outlines the processing algorithm and the optical/electrical assembly, and finally presents the results of the outdoor experiments.

\section{Lunar Landing Using Bio-Inspired Measurements}

Here, the principle of the control problem studied in this work is introduced. The landing scenario is presented along with initial and final states constraints. Then, the future approach of the biologically inspired feedback control loops is discussed.

\subsection{Autonomous Lunar Landing Strategy}

Lunar landing trajectory has been divided into four different phases in [15] (see Fig. 1):

1. De-orbit Phase,

2. Approach Phase,

3. Final Descent,

4. Free Fall. 
In this research work, a solution of the autonomy problem of the approach phase defined from the high gate (1800 $\mathrm{m} \pm 10 \%$ Above Ground Level -AGL-) to the low gate (10 m AGL) is studied. High gate corresponds to the height from which the landing site becomes visible from the spacecraft vision system. Low gate corresponds to the height from which the visual contact with the landing site is no longer available due to the dust raised by the thrusters. Initial parameters are a horizontal speed $V_{x_{0}}=69 \pm .03 \mathrm{~m} / \mathrm{s}$, a vertical speed $V_{z_{0}}=-36 \pm .03 \mathrm{~m} / \mathrm{s}$, a pitch angle $\theta_{0}=-61^{\circ}$, a ground height $h_{0}=1800 \pm 180 \mathrm{~m}$ and a mass $m_{l d r_{0}}=762 \pm 11 \mathrm{~kg}$ (see Fig. 1). This reference trajectory is thus very similar to the Apollo test case

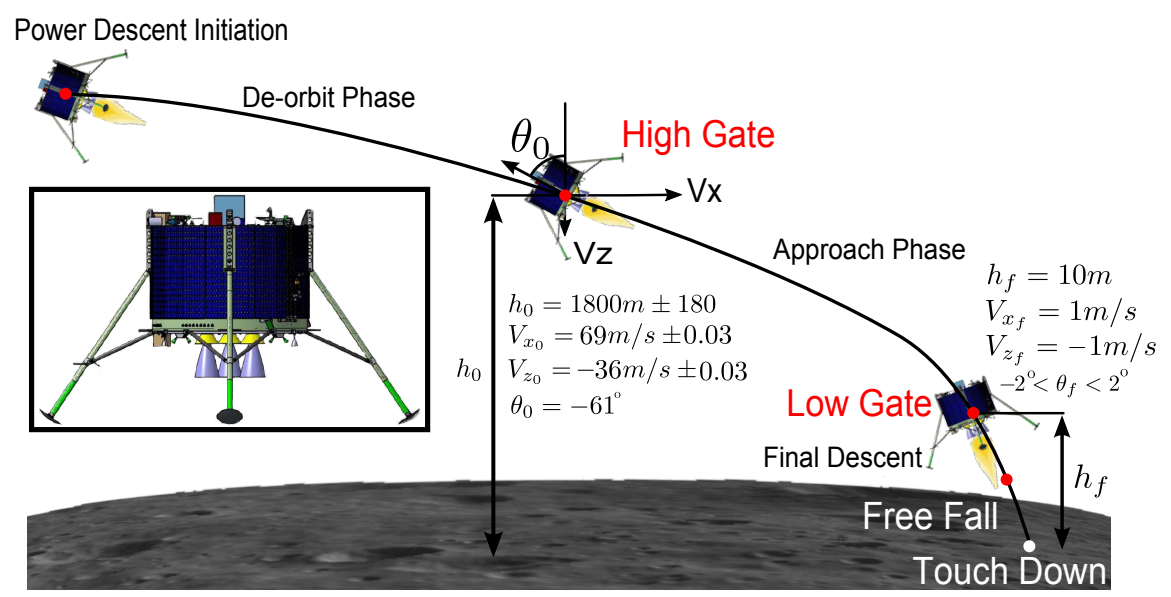

Fig. 1 Reference trajectory for lunar landing and 3D representation of the lunar module (courtesy: Astrium). The landing part addressed in this work is the approach phase defined between the high gate $(1800 \mathrm{~m} \pm 10 \% \mathrm{AGL})$ and the low gate $(10 \mathrm{~m} \mathrm{AGL})$. The objectives of the lander is to reach the low gate (10 m high) with both vertical and horizontal velocities lower than $1 \mathrm{~m} / \mathrm{s}$ and a pitch angle in the range $\pm 2^{\circ}$. Modified from [26].

scenario used in $[23,24,51]$. The considered solution features demanding terminal constraints at the low gate $\left(h_{f}=10 \mathrm{~m}\right)$ which are the following:

- $V_{x_{f}} \leq 1 \mathrm{~m} / \mathrm{s}$,

- $V_{z_{f}} \geq-1 \mathrm{~m} / \mathrm{s}$,

- $\left|\theta_{f}\right|<2^{\circ}$.

We do not introduce position accuracy since the intended landing strategy is not aiming at a pinpoint or even at a precision landing but only at a soft landing. The objectives are thus listed in terms of velocity and attitude. The position on the $\mathrm{x}$ axis is left free and concerning the altitude, the low gate will eventually be reached since the vertical velocity stays negative. In the current approach, the propellant mass consumption should be decrease as much as possible by the autonomous lunar landing strategy. The main challenge is that the entire state vector is not available from the measurements. For instance, velocities and position are neither measured 
nor estimated, only accelerations, angular rates, attitude, mass and optic flow are measured and thus available to feed the controllers. To land softly on the moon the autopilot should be able to reduce the velocity vector magnitude and to control the velocity vector orientation. This could be achieved by acting jointly on the lander's pitch and the lander's main thrust, the two available control signals. In [24, 51], authors have shown the substance of the pitch control law in the design to achieve optimal performances since the system is underactuated. In the solution currently under investigation, the main idea is to design an autopilot that keeps the main thrust antiparallel to the velocity vector orientation, in order to minimize fuel consumption. This principle states the pitch angle of reference $\theta_{\text {ref }}$ to be fed into the controller:

$$
\theta_{\text {ref }}=-\gamma-\frac{\pi}{2}
$$

Where $\gamma$ denotes the flight path angle (angle between the orientation of the speed vector and the local horizontal) as described in Fig. 2.

\subsection{Lander's Dynamic Modeling and Optic Flow Equations}

The autopilot under consideration consists mainly of an optic flow-based control system operating in the vertical plane $\left(e_{x}, e_{z}\right)$, which control the spacecraft's main thruster force and pitch angle. To stabilize the lander, it is necessary to cope with non-linearities and the inherent instability. Since there is no atmosphere on the

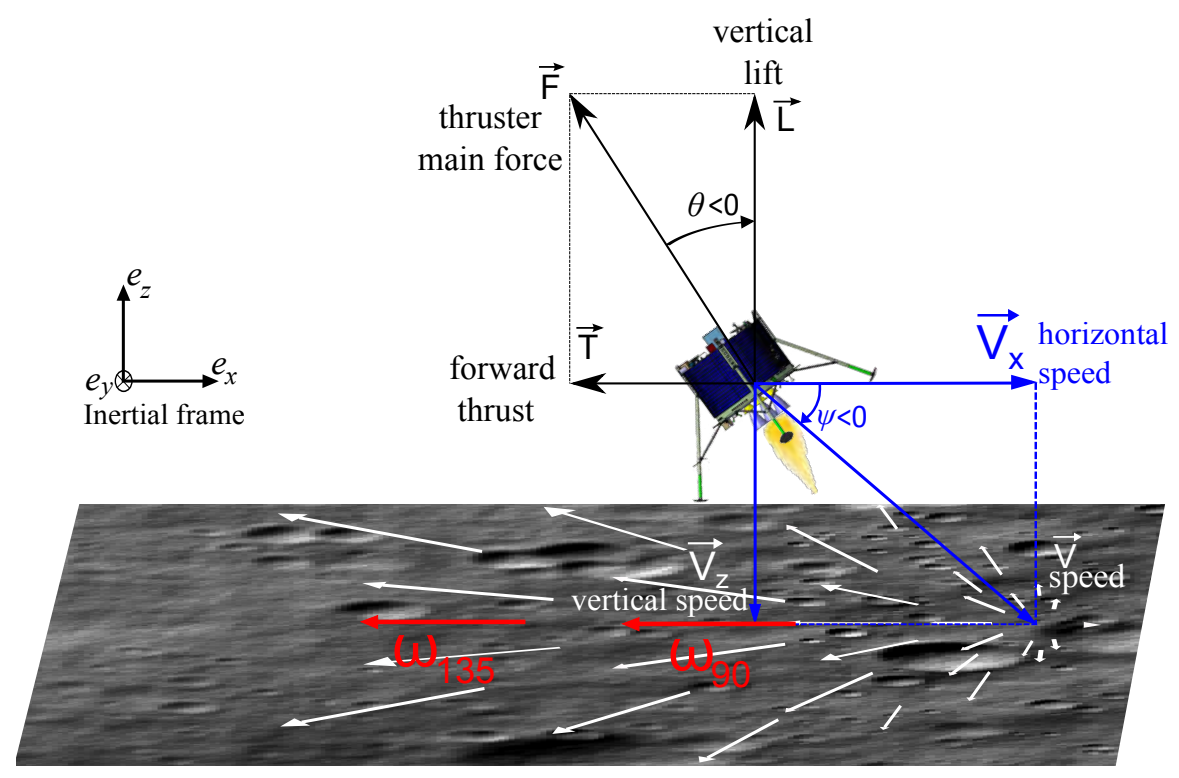

Fig. 2 Diagram of the lander, showing its speed vector $V$ and the mean thruster force $F$ 
Moon: no friction, wind or drag forces are applied to the lander. In the present model, the heave and surge dynamics are coupled via the lander's pitch (see Fig. 2). It is worth noting that it is not suitable using the scenario described in Fig. 1 to measure $\omega_{45}$ (where the subscript denotes the elevation angle: the angle between the gaze direction and the local horizontal) to determine the direction of the velocity vector, since it is near the focus of expansion where the motion is always null (see Fig. 2). To include physical constraints into the model, the following assumption is adopted:

(H1) $\left\{\begin{array}{l}\text { The thrusters can produce only positive forces and the maximum } \\ \text { thrust is limited to } 3820 \mathrm{~N} \text {, which means } 0 \leq u_{t h} \leq 3820 \mathrm{~N}\end{array}\right.$

The dynamic motion of the lander can be described in the time domain by the following dynamic system in the inertial frame $\left(e_{x}, e_{y}, e_{z}\right)$ :

$$
\left\{\begin{array}{l}
a_{l d r_{z}}(t)=\frac{\cos (\theta(t))}{m_{l d r}(t)} u_{t h}(t)-g_{\text {Moon }} \\
a_{l d r_{x}}(t)=\frac{\sin (\theta(t))}{m_{l d r}(t)} u_{t h}(t)
\end{array}\right.
$$

Where $u_{t h}=\|F\|$ corresponds to the control force applied to the lander, $a_{l d r_{x, z}}$ are the lander's accelerations in the lunar inertial reference frame, $m_{l d r}$ stands for the lander's mass, $\theta$ is the pitch angle, $t$ denotes the time and $g_{M o o n}$ the lunar gravity constant $\left(g_{\text {Moon }}=1.63 \mathrm{~m} / \mathrm{s}^{2}\right)$. The lander's mass depends directly on the fuel consumption, as given by the following relation:

$$
\dot{m}_{l d r}=\frac{-1}{I_{s p} \cdot g_{\text {Earth }}} u_{t h}(t)
$$

where $I_{s p}=311 \mathrm{~s}$ corresponds to the specific impulse and $g_{\text {Earth }}=9.81 \mathrm{~m} / \mathrm{s}^{2}$ to the Earth's gravity. This means that:

$$
m_{l d r}(t)=m_{l d r}\left(t_{0}\right)-\frac{1}{I_{s p} \cdot g_{E a r t h}} \int_{t_{0}}^{t} u_{t h}(\varepsilon) \mathrm{d} \varepsilon
$$

where $m_{l d r}\left(t_{0}\right)=762 \mathrm{~kg}$ is the lander's mass at high gate level. Since the initial mass is known and the lander's mass depends linearly on the integral of the lander's thruster control signal, the mass can be computed and assessed at any time during the simulated descent. The inner pitch control system is modeled as follows:

$$
\frac{I}{R} \frac{\mathrm{d}^{2} \theta}{\mathrm{d} t^{2}}=u_{\text {pitch }}(t)
$$

$u_{\text {pitch }}$ is the control input signal acting on the spacecraft's pitch, $\theta$ is measured via an IMU, $I$ the moment of inertia of the lander and $R$ the eccentricity of the thrusters from the center of mass. In this study, the well-known problem of error growth in IMU is not taken into account, the attitude measurement are thus considered accu- 
rate all along the descent. Once the dynamic model of the spacecraft is defined, one need to state the optic flow equations to find what information can be deduced from this visual cue. The ground-truth optic flow $\omega_{\text {grd-trh }}$ can be described as the sum of the two distinct components defined by [29], i.e. the translational and rotational optic flow:

$$
\omega_{g r d-t r h}=\omega_{T}+\omega_{R}
$$

The translational optic flow $\omega_{T}$ depends on the linear velocity $V$ expressed in the inertial frame associated with the vector basis $\left(e_{x}, e_{y}, e_{z}\right)$, the distance from the ground $D$ in the gaze direction and $\Phi$, the angle between the gaze direction and the heading direction.

$$
\omega_{T}=\frac{V}{D} \sin (\Phi)
$$

The rotational optic flow $\omega_{R}$ depends only on the angular speed $\Omega_{j}$ expressed in the body fixed frame, where $j$ denotes the axis of rotation, and on the elevation angle $\lambda$ between the gaze direction and the axis of rotation which is always $\frac{\pi}{2}$ in the $2 \mathrm{D}$ case (see [43] for a graphical illustration).

$$
\omega_{R}=\Omega_{j} \sin (\lambda)
$$

Finally the general equation of the optic flow is as follows:

$$
\omega_{\text {grd }-t r h}=\frac{V}{D} \sin (\Phi)+\Omega_{j} \sin (\lambda)
$$

From the previous equation and regarding hazardous terrain avoidance, one can see that as soon as an obstacle appears in the line of sight of the sensor, the distance $D$ will be reduced/increased whether it is a boulder or a crater. This will cause the autopilot to react by decelerating or by accelerating accordingly ensuring the safety of the lander no matter the topography of the terrain.

\subsection{Optic Flow Measurements $\omega_{90}$ and $\omega_{135}$ for Velocity Vector Orientation Estimation and Control}

In order to be able to strongly reduce the lander's speed during the approach phase, one needs to act both on the pitch angle and on the magnitude of the thrust as explained in Sect. 2.1. Since $\gamma$ is required for the pitch angle reference signal $\theta_{\text {ref }}$ generation and is not measured, one needs to find a way to estimate it by the means of the available sensors such as optic flow sensors and IMU. The main question is how to fuse different visual angular speed measurements to obtain useful information about unavailable measurements of the state vector. It is straightforward to note that the optic flow cue is related to the orientation of the velocity vector. Therefore, under the assumption that the sensors are embedded on a gimbal system one can derive the orientation angle $\gamma$ from optic flow sensors positioned in different directions. From 
(9), under the assumption of a practically flat ground (i.e. $D=h / \cos \left(\frac{\pi}{2}-\Phi+\gamma\right)$, $-\Phi+\gamma$ denotes the angle between the gaze direction and the local horizontal), and gimbaled mounted sensors (i.e. the camera rotation with respect to an inertial reference frame is kept to zero thanks to the attitude measurement provided by the IMU):

$$
\omega_{90}=\frac{V_{x}}{h}
$$

with $V=V_{x} / \cos (\gamma)$ and $h$ the ground height,

$$
\omega_{135}=\frac{V}{h / \cos (\pi / 4)} \frac{\sqrt{2}}{2}(\cos (\gamma)-\sin (\gamma))=\frac{\omega_{90}}{2}(1-\tan (\gamma))
$$

Finally one can obtain:

$$
\tan (\gamma)=1-2 \frac{\omega_{135}}{\omega_{90}}
$$

It is worth noting that both the horizontal and vertical dynamics are expressed in $\tan (\gamma)$ with $\tan (\gamma)=\frac{V_{z}}{V_{x}}$. Thanks to (12) featuring only visual information, a pitch controller based on optic flow and pitch measurement $\theta$ provided by the IMU could be designed through (5) in order to ensure the collinearity between the lander's main thruster force and its velocity vector orientation. The low speed visual motion sensors are thus the cornerstones of this autonomous lunar landing strategy. Since the optic flow controller and the pitch controller are based on the output signals of the optic flow sensors, it seemed to be worth testing the reliability of this sensor in real-life conditions. This is the purpose of the following section.

\section{VMS-Based Optic Flow Measurements Obtained Onboard ReSSAC}

As presented in the previous section, the control signals (pitch and thrust) which depend mainly on the optic flow measurement are strongly linked to the precision, robustness, sensitivity of the low speed visual motion sensors. That is why we had to develop and test a new VMS dedicated to angular speed measurements that cope with the range experienced during lunar landing, which is a range of low optic flow. Conveniently, the optic flow range experienced during lunar landing corresponds roughly to the range experienced with a high scale helicopter UAV which has been used to test the sensor on Earth.

\subsection{Bio-Inspired Optic Flow Processing}

A mandatory step in the maturation of a technology is to design and embed the previously simulated device on a real-life complex system. In order to validate the 
feasibility of the work performed in simulation using low speed VMS in Sect. 2, an experimental approach is presented. A low resolution visual motion sensor based on six pixels array and dedicated to low speeds has been developed to demonstrate the feasibility of measuring the 1-D local angular speed on a lunar landing like scenario on Earth. We tested the sensor onboard the ReSSAC unmanned helicopter to validate the bio-inspired algorithm on such low speeds at high heights and with strong disturbances (i.e. helicopter vibrations, uncontrolled illuminance, rough terrain). This sensor is an updated version of the 2-pixels Local Motion Sensors based on neuromorphic findings [12] designed in our laboratory.

\subsection{Presentation of the Low-Speed Visual Motion Sensor}

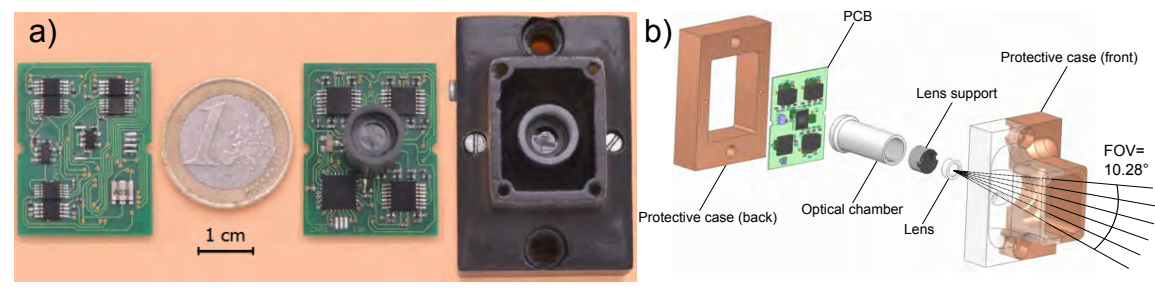

Fig. 3 (a) Top and bottom view of the electronic board (size: $33 \times 40 \mathrm{~mm}$ ) of a low-speed visual motion sensor with its lens mounted on the LSC photosensor array. The custom-made protective case is presented on the right. (b) Exploded view of the complete assembly, including the custommade protective case (front and back), the electronic board, and the optical assembly (lens, lens support, optical chamber).

The new low-speed visual motion sensor consists mainly of a low-cost plastic lens placed in front of an off-the-shelf photosensor array. The photosensor used in this study, which is called the LSC, was purchased from iC-Haus: it features six photodiodes, each having a large sensitive area of $300 \times 1600 \mu \mathrm{m}$ and an integrated preamplifier. The LSC conveys the visual signals received to a hybrid analog/digital processing algorithm, where the optic flow value $\omega_{\text {meas }}$ is computed. The cheap, lightweight lens used here was a CAX183 from Thorlabs (focal length $18.33 \mathrm{~mm}$, f-number 4.07). A custom-made protective case was added in order to protect the low-weight sensor and the optical assembly from unfavorable weather conditions (see Fig. 3.a for pictures and Fig. 3.b for an exploded view). The new visual motion sensor and its custom-made protective case weighed $29.4 \mathrm{~g}$. Many of the parameters of the original visual motion detecting scheme presented in $[5,39]$ have been updated, especially in terms of the optical angles and the cut-off frequency of the temporal filters. The six optical axes formed by the photodiodes are separated by an interreceptor angle $\Delta \varphi$ see Fig. 4. By defocusing the lens (i.e., by adjusting the distance between the lens and the photosensors), we obtained Gaussian angular sensitivity functions for each photoreceptor with a correlation coefficient greater than 


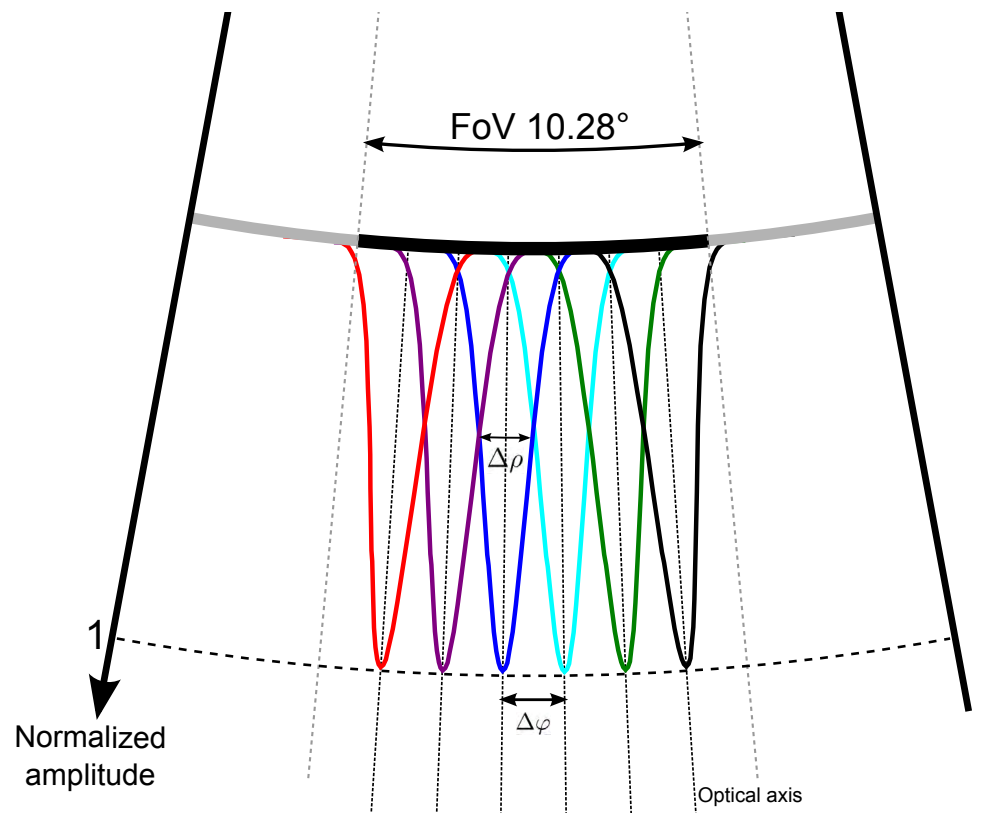

Fig. 4 Approximate Gaussian angular sensitivity functions of the LSC photosensor array with a CAX183 plastic aspherical lens. By adjusting the distance between the lens and the LSC photosensor, we obtained a correlation coefficient almost equal to $1\left(R_{L S C}^{2}>0.990\right)$, and a $\Delta \varphi$ value approximately equal to $\Delta \rho$.

99\% ( $\left.R_{L S C}^{2}>0.990\right)$, in line with what occurs in the common fly's eye [17]. These features were assessed by slowly rotating the lens in front of a point light source placed at a distance of $85 \mathrm{~cm}$. The local 1-D angular speed $\omega_{\text {meas }}$ measured by the sensor was defined as the ratio between the interreceptor angle $\Delta \varphi$ and the time elapsing $\Delta t$ between the moments when two adjacent photodiode signals reach the threshold (i.e., the time of travel of a contrast from the optical axis of one photodiode to the optical axis of the following one).

$$
\omega_{\text {meas }}=\frac{\Delta \varphi}{\Delta t}
$$

In [10], the measurement range of the sensor covered a large range of high speeds from $50^{\circ} / \mathrm{s}$ to $300^{\circ} / \mathrm{s}$, whereas the present study focused on low velocities giving a range of $1.5^{\circ} / \mathrm{s}$ to $25^{\circ} / \mathrm{s}$, which is more than tenfold slower. In order to stay in the same range of $\Delta t$, whose accuracy of measurement depends on the microcontroller's sampling frequency, we therefore had to narrow $\Delta \varphi . \Delta \varphi$ corresponds to the angle separating two adjacent photodiodes optical axis: it depends on the focal lens, on the pitch (distance between the center of two adjacent photodiodes) and also on the distance from the photodiode plane to the focal point which is the easiest setting to adjust. The large $18.33 \mathrm{~mm}$ focal length increases the defocalizing effects of the 
lens, giving a suitably small mean interreceptor angle of $\overline{\Delta \varphi}=1.488^{\circ}$. The second advantage of the defocusing process is that it adds a blurring effect giving Gaussianshaped angular sensitivity functions. As found to occur in some diurnal insects [30],

$$
\Delta \varphi=\Delta \rho
$$

Achieving a tight $\Delta \rho$ made it possible for the sensor to respond to higher spatial frequency contrasts. The acceptance angle, defined by $\Delta \rho$, acts like an optical low pass spatial filter. We eventually reached $1.4^{\circ} \geq \Delta \varphi \approx \Delta \rho<1.5^{\circ}$, corresponding to a field of view in the direction of the visual motion sensor of $10.28^{\circ}$ $\left(\sum_{i=1}^{5} \Delta \varphi_{i}+\frac{\Delta \varphi_{1}}{2}+\frac{\Delta \varphi_{5}}{2}\right)$. Table 1 gives the optical characteristics of the sensor. The

Table 1 Characteristics of the new low-speed visual motion sensor

\begin{tabular}{ll}
\hline Parameter & Value \\
\hline Focal length of the lens CAX183 $(\mathrm{mm})$ & 18.33 \\
$f_{\text {number }}$ of the lens $(\#)$ & 4.07 \\
Angular velocity range $\left({ }^{\circ} / \mathrm{s}\right)$ & {$[1.5 ; 25]$} \\
Field of view of a single photodiode $\left(^{\circ}\right)$ & $2.90 \times 15.44$ \\
Sensitivity $\left({ }^{\circ} / \mathrm{s} / \mathrm{LSB}\right)$ & $4.58 \mathrm{e}-5$ \\
Mean interreceptor angle $\overline{\Delta \varphi}\left({ }^{\circ}\right)$ & 1.488 \\
Mean acceptance angle $\overline{\Delta \rho}\left({ }^{\circ}\right)$ & 1.448 \\
Photodiode size $(\mu \mathrm{m})$ & $300 \times 1,600$ \\
Pixel pitch $(\mu \mathrm{m})$ & 420 \\
Resolution $\left({ }^{\circ} / \mathrm{s}\right)[\mathrm{min} ; \max ]$ & {$[0.01 ; 0.21]$} \\
Mass of the visual motion sensor in a stand-alone version $(\mathrm{g})$ & 2,8 \\
\hline
\end{tabular}

general processing algorithm consists of two parts: an analog processing part converts the six visual signals into electrical signals with a high signal to noise ratio, and the digital processing part then simultaneously computes five optic flow values plus the median value (see Fig. 5). The analog processing begins with a programmable gain connected to the microcontroller via a SPI communication bus [46]. A passband filter then differentiates the visual signal and acts as an anti-aliasing filter. The digital processing algorithm starts with a second order fixed-point notch filter centered on the ReSSAC's main rotor frequency. The center frequency of the filter is $f_{0}=13.8 \mathrm{~Hz}$ with a Q-factor $Q=6.9$ at a sampling frequency $f_{s}=500 \mathrm{~Hz}$. Its transfer function, which has been defined in [35], is as follows:

$$
H_{\text {notch }}(z)=b \frac{1-2 \cos \left(\omega_{0}\right) z^{-1}+z^{-2}}{1-2 b \cos \left(\omega_{0}\right) z^{-1}+(2 b-1) z^{-2}}
$$

with

$$
b=\frac{1}{1+\frac{\sqrt{1-G_{B}^{2}}}{G_{B}} \tan \left(\frac{\Delta \omega}{2}\right)}
$$




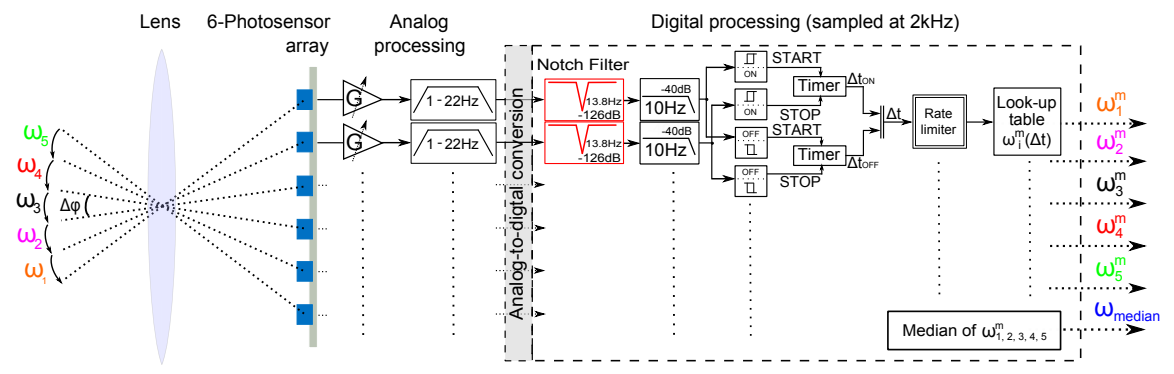

Fig. 5 General processing architecture of the low-speed visual motion sensor. First of all, the spatial sampling and low-pass filtering steps are carried out by the defocused lens. The six photodiode signals are amplified by a programmable gain in order to increase the signal to noise ratio, before being filtered by an analog bandpass filter $(1-22 \mathrm{~Hz})$. The digital stage begins with a second order fixed-point notch filter centered on the main rotor frequency of ReSSAC, 13.8 Hz. It is followed by a second order fixed-point low pass filter with a cut-off frequency set at $10 \mathrm{~Hz}$. A hysteresis thresholding process is associated with the computation of the time $\Delta t$ elapsing between two adjacent signals (with either ON or OFF contrasts). Lastly, after an outlier filtering step, the output signal of the 1-D visual motion sensor is obtained from a precomputed look-up table and the median value is calculated.

where $\Delta \omega$ is the full width at a level $G_{B}^{2}$ and $\omega_{0}$ is the center frequency. We chose $\omega_{0}=2 \pi \frac{f_{s}}{f_{0}}, \Delta \omega=2 \pi \frac{\Delta f}{f_{s}}$ with $\Delta f=2 \mathrm{~Hz}$ and $G_{B}^{2}=-3 d B$. As the visual angular speed $\omega_{\text {meas }}$ is quite low, the temporal frequency $f_{t}$ of the visual signal (which consists of contrasts) is also quite low, as expressed by the following equation [31]:

$$
f_{t}=\omega_{\text {meas }} \times f_{\text {spatial }}
$$

where $f_{\text {spatial }}$ is the spatial frequency (in cycles $/^{\circ}$ ) associated with the contrasting pattern.

Therefore, a second order fixed-point low pass filter was used to enhance the signal to noise ratio by removing the noise remaining at frequencies of more than $10 \mathrm{~Hz}$. The algorithm called the "Time of travel scheme" implemented here consists mainly of a hysteresis thresholding process with separate ON and OFF pathways $[5,39,40,42,45]$ followed by the $\Delta t$ computation, the result of which is fed into a corresponding table. Lastly, the five simultaneously computed optic flows $\omega_{i}^{m}$ are combined by the median operator in order to increase the robustness and the refresh rate of the output [40]. The microcontroller used for this purpose is a dsPIC33FJ128GP802 working at a sampling frequency of $2 \mathrm{kHz}$, except for the digital filters, which are sampled at a rate of $500 \mathrm{~Hz}$. Special efforts were made to optimize the algorithm, and a computational load of only $17 \%$ was eventually obtained. 


\subsection{Free-Flying Results with ReSSAC}

The characteristics of the present visual motion sensor (VMS) were assessed by performing optic flow measurements under controlled motion conditions (orientation and velocity) outdoors. Pure rotational motion was applied to the sensor with angular speed variations ranging from $1^{\circ} / \mathrm{s}$ to $20^{\circ} / \mathrm{s}$ using a previously described outdoor set-up [10]. The triangular response pattern obtained corresponds closely to the reference angular speed (see Fig. 6). It can therefore be said that this new tiny sensor is able to accurately compute the 1-D visual angular speed during a rotational motion within its operating range. The refresh rate is defined as the ratio between the total number of new measurements of each $\omega_{i}$ occurring within the acceptable range $\left[1.5^{\circ} / \mathrm{s}-25^{\circ} / \mathrm{s}\right]$ and the time elapsing. The median value is delivered at $2 \mathrm{kHz}$ (output data rate) even if the measure is not refreshed, that is why the refresh rate metric is needed to evaluate the performance of the sensor. The mean refresh rate achieved during the dynamic performances evaluation was $f_{\text {refresh }}=6.64 \mathrm{~Hz}$ : this value depends on the richness of the visual environment, as well as on the actual angular speed. The low-speed VMS performances were then studied on a six degrees

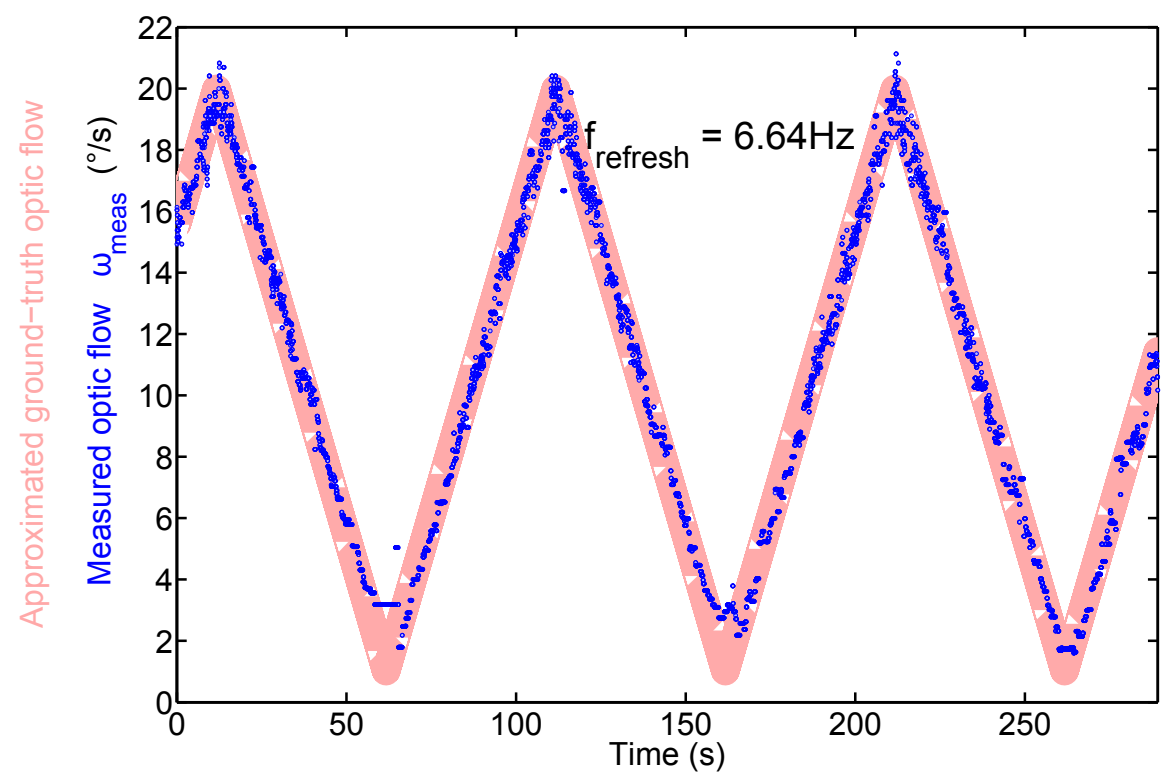

Fig. 6 Dynamic outdoor response of the low-speed VMS (blue), as compared with the groundtruth optic flow (red). The visual motion sensor was rotated by means of a conveyor belt driven by a stepping motor (103H5208-0440 from Sanyo-Denki) [10]. Rotations from $1^{\circ} / \mathrm{s}$ to $20^{\circ} / \mathrm{s}$ were applied to the sensor, which is designed to operate in the $1.5^{\circ} / \mathrm{s}$ to $25^{\circ} / \mathrm{s}$ range. The optic flow measured closely matched the reference signal, with a refresh rate of $6.64 \mathrm{~Hz}$. Since no synchronization signal was available, the ground-truth optic flow has been roughly synchronized here. 
of freedom UAV during free flight over fields. The ONERA's ReSSAC unmanned helicopter was used to test the sensor's dynamic responses. The characteristics of ReSSAC (Yamaha RmaX) in terms of the mass balance have been described in [53]. Its mass, its flight envelope and the vibration dynamics due to the main rotor's rotational speed presented us with quite a challenging ground-truth optic flow profile. The flight was performed in South-western France in mid-July around 5pm on a bright sunny day: the mean illuminance was approximately $10000 \mathrm{~lx}$. Another tests should be performed on special setup at ESA or Astrium facilities to be sure that the sensor would respond similarly in a moon-like environment in terms of robustness to moon-like contrasts and illuminance. Onboard ReSSAC, the 1-D local optic flow measured is subject to several variations as follows. Since the roll and pitch angles are small during the whole flight, the distance to the ground in the gaze direction $D$ can be approximated as $D \approx h /(\cos (\varphi) \cos (\theta))$, where $\varphi$ denotes the roll angle, $\theta$ denotes the pitch angle and $h$ denotes the local ground height. In our case, $\Phi=-\theta+\gamma+\frac{\pi}{2}$ (with the sensor oriented downward, $\gamma<0, \theta<0$ ), $\lambda=\frac{\pi}{2}$ and $\Omega_{j}=\Omega_{2}$, where $\Omega_{2}$ is the pitch angular velocity defined in the body fixed reference frame, the ground-truth optic flow (see (9)) is therefore computed as described in (17).

$$
\omega_{\text {grd }-t r h}=\left(\frac{V}{h} \cos (\theta) \cos (\varphi) \sin \left(\theta+\gamma+\frac{\pi}{2}\right)\right)+\Omega_{2}
$$

During the experiment described below, the approximate ground-truth optic flow $\omega_{\text {grd-trh }}$ was computed using data from the IMU, the GPS (OEM4 G2 from NovAtel) and the data grabbed by a LIDAR (Sick LDMRS 400001) during previous flights over the same fields. We computed the ground-truth optic flow as precisely as possible but since the data are coming from cartographic data previously recorded and from different sensors with different accuracy as well as different noise sources: $\omega_{\text {grd-trh }}$ is the approximate ground-truth optic flow. The low speed visual motion sensor was embedded at the front end of ReSSAC pointing directly downward with a clear field of view. Fig. 7 shows the nice response of the low-speed visual motion sensor mounted onboard the unmanned ReSSAC helicopter. Despite the complex ground-truth optic flow, the visual motion sensor responded appropriately to the visual stimuli. The standard deviation of the error between the ground-truth optic flow $\omega_{\text {grd-trh }}$ and the measured optic flow $\omega_{\text {meas }}$ was less than $2.25^{\circ} / \mathrm{s}$, which is quite low. The refresh rate $f_{\text {refresh }}$ was greater than $7.8 \mathrm{~Hz}$, which is even slightly higher than in the dynamic measurements performed during a rotating motion on ground. Fig. 7.b, giving the pitch angular rate of ReSSAC and Fig. 7.c the local ground height shows how well the sensor responded to its visual environment and how complex is the visual motion combining at the same time erratic relief and pitch variations. Once again, the low-speed VMS accurately sensed these height variations and yielded similar values to the ground-truth value. The robust and accurate performances observed during this experiment show that the low-speed visual motion sensor is highly suitable for use in many high-scaled robotic applications. 


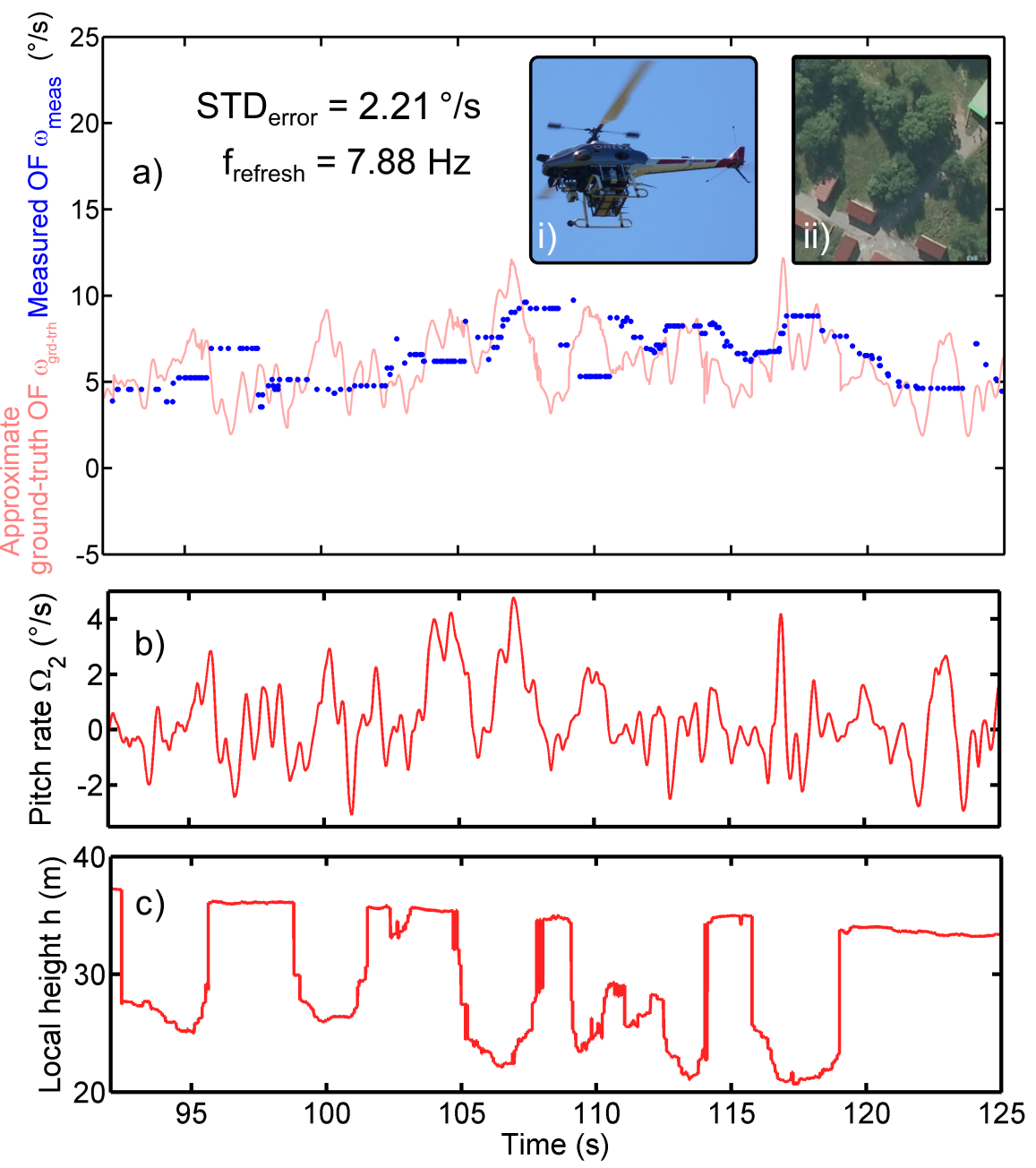

Fig. 7 Low-speed visual motion sensor and flight data sensed on-board the ReSSAC UAV. (a) Approximate ground-truth optic flow (see (17)) (red) and measured optic flow $\omega_{\text {meas }}=\Delta \varphi / \Delta t$ (blue dots). Despite the strong variations mainly due to vibrations, the low-speed visual motion sensor's output closely matched the approximate ground-truth optic flow, giving a standard deviation of $2.21^{\circ} \mathrm{s}$ and a refresh rate of $7.88 \mathrm{~Hz}$. The effects of strong variations in the local height due to the successive trees and houses are directly reflected in the low-speed VMS measurement signal. (i) ReSSAC unmanned helicopter in-flight (ii) Aerial view of the flight environment obtained on http://geoportail.fr. (b) Pitch angular rate of ReSSAC as measured by the IMU. (c) Local ground height measured by combining GPS data and previously mapped LIDAR data. The nominal height was around $40 \mathrm{~m}$. But due to the variable relief, the local height often changed suddenly by 15 meters. 


\section{Conclusion}

In this paper, we gathered several emerging technologies to achieve an autonomous lunar landing based on low-speed optic flow sensors. The mathematical expression describing the dynamic model of the spacecraft as well as the optic flow fusion allowing some useful states estimation have been presented along with the intended control strategy based on two parallel control loops acting on the lander's pitch angle and main thruster. The main idea of this promising biologically inspired approach is to avoid the direct measurement of the height or velocity. We intend to design the thrust control law using optic flow measurements while keeping the main thruster force collinear to the velocity vector in a concern of mass consumption optimization. We showed that with a sensor set-up based on two optic flow sensors pointing downward in different directions $\left(90^{\circ}\right.$ and $135^{\circ}$ from the local horizontal), we obtained a direct measurement of $\gamma$ the orientation of the velocity vector allowing us to generate a reference signal for the pitch angle controller. The next step will be to develop the full simulations featuring two control loops to achieve at low gate the final conditions stated in the reference scenario presented in this paper. The loop controlling the main thruster will be designed using optic flow cue. Secondly the gimbal setup used in simulations needs to be addressed. Since the main benefits of this minimalistic optic flow technology is the weight efficiency and simplicity, a gimbal system is then not suited for this purpose. Increasing the number of VMS and thus enlarging the sensory field of view is a potential way to achieve such challenge. The extension of the solution to a real-life, 3D setup, is an interesting objective to be addressed. Furthermore, during the flight on ReSSAC, even if the yaw and roll angle are considered small, they exist during the whole flight without deteriorating the measurements performances. Once the strategy of the lunar landing was introduced, we presented a new lightweight visual motion sensor able to compute accurately the optic flow in the range experienced during a quasioptimal approach phase of a lunar landing. This new VMS has been developed, and then tested both on the ground and in flight onboard a $80 \mathrm{~kg}$ unmanned helicopter called ReSSAC over an unknown complex outdoor environment and under real-life dynamic and vibratory conditions. Encouraging results of this experiment showed that this sensor is perfectly suited for aeronautics or aerospace applications since it sensed accurately the local $1-\mathrm{D}$ angular speed ranging from $1.5^{\circ} / \mathrm{s}$ to $25^{\circ} / \mathrm{s}$ with a quite frequently refreshed measurement. In future work emphasis will be placed on the lunar landing simulations using Matlab/Simulink(c) and the PANGU software using the $\tan (\gamma)$ estimation method and the control scheme presented here.

Acknowledgements We are most grateful to N. Franceschini, S. Viollet, G. Graton, F. Roubieu and F. Expert for their fruitful suggestions and comments during this study. We thank P. Mouyon, H. de Plinval, A. Amiez and V. Fuertes for the successful flight of ReSSAC, T. Rakotomamonjy and A. Piquereau for their expertise in helicopter dynamics, M. Boyron for his assistance with the electrical design and J. Diperi for his involvement in the mechanical design. We would like to thank the two anonymous referees for providing us with constructive comments and suggestions. This research work is co-funded by European Space Agency and Astrium Satellites under ESA's Networking/Partnering Initiative program (NPI) for advanced technologies for space. 


\section{References}

1. Barrows, G., Neely, C.: Mixed-mode VLSI optic flow sensors for in-flight control of a Micro Air Vehicle. In: SPIE: Critical technologies for the future of computing, 52-63 (2000)

2. Benson, R., Delbrück, T., Touretzky, D. S.: Direction selective silicon retina that uses null inhibition. In: Adv. Neural Inf. Process. Syst. 4, ed. Morgan Kaufman, San Mateo, CA, 756763 (1992)

3. Beyeler, A., Zufferey, J., Floreano, D.: OptiPilot: control of take-off and landing using optic flow, In: European Micro Aerial Vehicle Conference (EMAV) (2009)

4. Beyeler, A., Zufferey, J. C., Floreano, D.: Vision-based control of near-obstacle flight. In: Auton. Robot. 27, 201-219 (2009)

5. Blanes, C.: Appareil Visuel élémentaire pour la navigation à vue d'un robot mobile autonome, Master's thesis (Advisor: N. Franceschini), Neurosciences, Univ. Aix-Marseille II (1986)

6. Braun, R., Manning, R.: Mars exploration entry, descent and landing challenges. In: IEEE Aerosp. Conf., Pasadena, CA : Jet Propulsion Laboratory, National Aeronautics and Space Administration, Big Sky, Montana (2006)

7. Cheng, Y., Ansar, A.: Landmark Based Position Estimation for Pinpoint Landing on Mars. In: IEEE Int. Conf. Robot. Autom. (ICRA), 1573-1578 (2005)

8. Conroy, J., Gremillion, G., Ranganathan, B., Humbert, J.: Implementation of wide-field integration of optic flow for autonomous quadrotor navigation. In: Auton. Robot. 27, 189-198 (2009)

9. Dubois-Matra, O., Parkes, S., Dunstam, M.: Testing and Validation of Planetary Vision-based navigation systems with PANGU. In: 21st International Symposium on Space Flight Dynamics (ISSFD) (2009)

10. Expert, F., Viollet, S., Ruffier, F.: Outdoor Field Performances of Insect-Based Visual Motion Sensors. In: J. Field Robot. 28, 529-541 (2011)

11. Flandin, G., Polle, B., Frapard, B., Vidal, P., Philippe, C., Voirin, T.: Vision Based Navigation for Planetary Exploration. In: 32nd Annual AAS Rocky Mountain Guidance and Control Conference (2009)

12. Franceschini, N., Riehle, A., Nestour, A.Stavenga, D., Hardie, R.: Directionally selective motion detection by insect neurons. In: Facets of vision, Springer, 360-390 (1989)

13. Franceschini, N., Pichon, J., Blanes, C.: From insect vision to robot vision. In: Philos. T. R. Soc. Lond. 337, 283-294 (1992)

14. Franceschini, N., Ruffier, F., Serres, J.: A Bio-Inspired Flying Robot Sheds Light on Insect Piloting Abilities. In: Curr. Biol. 17, 329-335 (2007)

15. Frapard, B., Champetier, C., Kemble, S., Parkinson, B., Strandmoe, S., M., L.: Vision-Based GNC Design for the LEDA Mission. In: ESA 3rd International Conference on Spacecraft GNC (1996)

16. Frapard, B., Polle, B., Flandin, G., Bernard, P., Vtel, C., Sembely, X., Mancuso, S.: Navigation for Planetary Approach and Landing. In: 5th International ESA Conference on GNC, pp. 159 (2002)

17. Götz, K.: Optomotorische untersuchung des visuellen systems einiger Augenmutanten der fruchtfliege Drosophila. In: Biol. Cybern. 2, 77-92 (1964)

18. Garratt, M., Chahl, J.: Vision-Based Terrain Following for an Unmanned Rotorcraft. In: J. Field Robot. 25, 284-301 (2008)

19. Green, W., Oh, P., Barrows, G.: Flying insect inspired vision for autonomous aerial robot maneuvers in near-earth environments. In: IEEE Int. Conf. Robot. Autom. (ICRA), 23472352 Vol.3 (2004)

20. Griffiths, S., Saunders, J., Curtis, A., Barber, B., McLain, T., Beard, R.: Maximizing miniature aerial vehicles. In: IEEE Robot. Autom. Mag. 13, 34-43 (2006)

21. Hérissé, B., Hamel, T., Mahony, R., Russotto, F.-X.: Landing a VTOL Unmanned Aerial Vehicle on a Moving Platform Using Optical Flow. In: IEEE T. Robot. 28(1), 77-89 (2012)

22. Hrabar, S., Sukhatme, G., Corke, P., Usher, K., Roberts, J.: Combined optic-flow and stereobased navigation of urban canyons for a UAV. In: IEEE Int. Conf. Intell. Rob. Syst. (IROS), 3309-3316 (2005) 
23. Izzo, D., de Croon, G.: Landing with time-to-contact and ventral optic flow estimates. In: J. Guid. Control Dynam. 35 (4), 1362-1367 (2011)

24. Izzo, D., Weiss, N., Seidl, T.: Constant-Optic-Flow Lunar Landing: Optimality and Guidance. In: J. Guid. Control Dynam. 34, 1383-1395 (2011)

25. Janschek, K., Tchernykh, V., Beck, M.: Performance Analysis for Visual Planetary Landing Navigation Using Optical Flow and DEM matching. In: AIAA Guidance, Navigation and Control Conference and Exhibit (2006)

26. Jean-Marius, T., Strandmoe, S. E.: Integrated vision and navigation for a planetary lander, ESA, Estec, Technical report, AEROSPATIAL, Espace et Dfense, Les Mureaux, France (1998)

27. Kendoul, F., Fantoni, I., Nonamib, K.: Optic flow-based vision system for autonomous 3D localization and control of small aerial vehicles. In: Robot. Auton. Syst. 57, 591-602 (2009)

28. Kendoul, F., Nonami, K., Fantoni, I., Lozano, R.: An adaptive vision-based autopilot for mini flying machines guidance, navigation and control. In: Auton. Robot. 27, 165-188 (2009)

29. Koenderink, J., Doorn, A.: Facts on optic flow. In: Biol. Cybern. 56, 247-254 (1987)

30. Land, M.: Visual Acuity In Insects. In: Annu. Rev. Entomol. 42, 147-177 (1997)

31. Landolt, A., Mitros, A.: Visual sensor with resolution enhancement by mechanical vibrations. In: Auton. Robot. 11 (3), 233-239 (2001)

32. Mahony, R., Corke, P., Hamel, T.: A Dynamic Image-Based Visual Servo Control Using Centroid and Optic Flow Features. In: J. Dyn. Sys., Meas., Control 130(1), 1-12 (2008)

33. Moeckel, R., Liu, S.-C.: Motion Detection Circuits for a Time-To-Travel Algorithm. In: IEEE Int. Symp. Circ. S. (), 3079-3082 (2007)

34. Mourikis, A. I., Trawny, N., Roumeliotis, S. I., Johnson, A. E., Ansar, A., Matthies, L.: VisionAided Inertial Navigation for Spacecraft Entry, Descent, and Landing. In: IEEE Trans. Robot. 25(2), 264-280 (2009)

35. Orfanidis, S.J.: Introduction to signal processing, ed. Prentice-Hall Inc., Upper Saddle River, NJ, USA (1995)

36. Parkes, S., Silva, V.: GNC sensors for planetary landers : a review. In: Data Systems in Aerospace (DASIA), 1-9 (2002)

37. Parkes, S., Dunstan, M., Matthews, D., Martin, I., Silva, V.: LIDAR-based GNC for Planetary Landing: Simulation with PANGU. In: Data Systems in Aerospace (DASIA), ed. R.A. Harris, Prague, Czech Republic, 18.1 (2003)

38. Parkes, S., Martin, I., Dunstan, M.: Planet Surface Simulation with PANGU. In: 8th International Conference on Space Operations, 1-10 (2004)

39. Pichon, J.-M., Blanes, C., Franceschini, N.: Visual guidance of a mobile robot equipped with a network of self-motion sensors. In: SPIE Conf. on Mobile Robots IV, ed. W.J. Wolfe and W.H. Chun, 44-53 (1989)

40. Roubieu, F., Expert, F., Boyron, M., Fuschlock, B., Viollet, S., Ruffier, F.: A novel 1-gram insect based device measuring visual motion along 5 optical directions. In: IEEE Sens. Conf., 687-690 (2011)

41. Roumeliotis, S., Johnson, A., Montgomery, J.: Augmenting inertial navigation with imagebased motion estimation. In: IEEE Int. Conf. Robot. Autom. (ICRA), 4326-4333 (2002)

42. Ruffier, F., Viollet, S., Amic, S., Franceschini, N.: Bio-inspired optical flow circuits for the visual guidance of micro air vehicles. In: IEEE Int. Symp. Circ. S. (ISCAS), 846-849 (2003)

43. Ruffier, F.: Pilote automatique biomimétique. Système générique inspiré du contrôle visuomoteur des insectes pour: le suivi de terrain, la réaction au vent et l'atterrissage automatiques d'un micro-aéronef. PhD thesis (in French), Institut National Polytechnique de Grenoble (2004)

44. Ruffier, F., Franceschini, N.: Visually guided micro-aerial vehicle: automatic take off, terrain following, landing and wind reaction. In: IEEE IEEE Int. Conf. Robot. Autom. (ICRA), 2339-2346 (2004)

45. Ruffier, F., Franceschini, N.: Optic flow regulation: the key to aircraft automatic guidance. In: Robot. Auton. Syst. 50, 177-194 (2005) 
46. Ruffier, F., Expert, F.: Visual motion sensing onboard a 50-g helicopter flying freely under complex VICON-lighting conditions. In: International Conference on Complex Medical Engineering, 634-639 (2012)

47. Shang, Y., Palmer, P.: The dynamic motion estimation of a lunar lander. In: 21t International Symposium on Space Flight Dynamics (ISSFD), 1-14 (2009)

48. Strandmoe, S., Jean-Marius, T., Trinh, S.: Toward a vision based autonomous planetary lander. In: AIAA Guidance, Navigation, and Control Conference and Exhibit, Portland, OR, AIAA-99-4154 (1999)

49. Tchernykh, V., Beck, M., Janschek, K.: An Embedded Optical Flow Processor for Visual Navigation using Optical Correlator Technology. In: IEEE Int. Conf. Intell. Rob. Syst. (IEEE/RSJ), Beijing, 67-72 (2006)

50. Trawny, N., Mourikis, A. I., Roumeliotis, S. I., Johnson, A. E., Montgomery, J.: Vision-aided inertial navigation for pin-point landing using observations of mapped landmarks. In: J. Field Robot. 24, 357-378 (2007)

51. Valette, F., Ruffier, F., Viollet, S., Seidl, T.: Biomimetic optic flow sensing applied to a lunar landing scenario. In: IEEE Int. Conf. Robot. Autom. (ICRA), 2253-2260 (2010)

52. Watanabe, Y., Fabiani, P., Le Besnerais, G.: Simultaneous visual target tracking and navigation in a GPS-denied environment. In: Int. Conf. Adv. Robot. (ICAR), 1-6 (2009)

53. Watanabe, Y., Lesire, C., Piquereau, A., Fabiani, P., Sanfourche, M., Le Besnerais, G.: The ONERA ReSSAC Unmanned Autonomous Helicopter: Visual Air-to-Ground Target Tracking in an Urban Environment. In: American Helicopter Society 66th Annual Forum (2010) 